\title{
Antitumor activity and pharmacokinetics of oral gimatecan on pediatric cancer xenografts
}

\author{
Massimo Zucchetti • Daniela Meco • Angela Maria Di Francesco • Tiziana Servidei • \\ Valentina Patriarca $\cdot$ Gabriella Cusano $\cdot$ Maurizio D'Incalci $\cdot$ \\ Daniele Forestieri $\cdot$ Claudio Pisano $\cdot$ Riccardo Riccardi
}

Received: 30 July 2009 / Accepted: 27 November 2009 / Published online: 20 December 2009

(C) Springer-Verlag 2009

\begin{abstract}
Purpose This study compared the antitumor activity and the pharmacological profile of gimatecan given orally and irinotecan (CPT-11) on pediatric tumor xenografts.

Experimental design Gimatecan was tested in two neuroblastoma cell lines (SK-N-DZ and SK-N-(BE)2c) and on TE-671 rhabdomyosarcoma cells using two different schedules. We characterized its pharmacokinetic profile in nude mice bearing human SK-N-DZ and TE-671 cell lines.

Results Gimatecan appears to have high plasma disposition. The drug was present in plasma almost completely as the intact lactone form and showed substantial activity in all tumor models. Prolonged daily treatment with low doses of gimatecan produced significant tumor regression in all tumor xenografts.

Conclusion The antitumor activity and the promising pharmacological profile indicate gimatecan as an excellent candidate for clinical treatment of pediatric tumors.
\end{abstract}

The authors Massimo Zucchetti and Daniela Meco contributed equally to the work.

M. Zucchetti $(\square) \cdot$ M. D’Incalci · D. Forestieri

Department of Oncology,

Istituto di Ricerche Farmacologiche "Mario Negri”,

Via La Masa 19, 20156 Milan, Italy

e-mail: zucchetti@marionegri.it

D. Meco - A. M. Di Francesco - T. Servidei - V. Patriarca .

G. Cusano $\cdot$ R. Riccardi

Division of Pediatric Oncology,

Catholic University of Rome, Largo A. Gemelli 8,

00168 Rome, Italy

C. Pisano

Research \& Development, Sigma-Tau, Rome, Italy
Keywords Gimatecan · Topoisomerase I - Camptothecin . Pediatric tumor xenografts $\cdot$ Pharmacokinetics

\section{Introduction}

Camptothecins are a class of antineoplastic agents with a wide spectrum of antitumor activity and are among the most effective drugs for the treatment of solid tumors. Their mechanism of action involves the inhibition of DNA topoisomerase I [1]. DNA topoisomerases are enzymes, which modify DNA topology by transiently breaking strands of the DNA, and are particularly important in replication and transcription. Camptothecin and its derivatives stabilize the cleavable complex between topoisomerase I and single-strand DNA that inhibits the DNA relegation [2].

Topotecan and irinotecan (CPT-11), which are derivatives of camptothecins, are nowadays regularly employed in clinical practice. CPT-11 is a water-soluble analog that has shown significant activity against a number of different tumor types in preclinical and in clinical trials of patients with colon, stomach, pancreas and non-small-cell lung cancers [3-5]. Moreover, it has been demonstrated that neuroblastoma xenografts are highly sensitive to CPT-11 [6]. The active metabolite of CPT-11, SN-38, stabilizes a ternary complex between the nuclear enzyme topoisomerase I and double-stranded DNA [7]. As reported by Santos et al., CPT-11 shows marked antitumoral activity against pediatric xenografts in preclinical studies $[8,9]$. Topotecan is used as a standard treatment in ovarian cancer, and it was recently reported to be effective in a small percentage of patients with relapsed or refractory neuroblastoma [10-12]. However, camptothecins suffer some problems in the pharmacological profile, such as reversibility of the drug-target 
interaction, the opening of the lactone ring and subsequent binding with serum albumin [13-16].

Recently, new camptothecins substituted at position 7 with lipophilic chains have been synthesized with the purpose to help the stabilization of the lactone form that is responsible for the pharmacological activity $[13,17]$. One of these, gimatecan (Fig. 1), is particularly interesting, because it offers strong cytotoxic potency, due to topoisomerase I inhibition, enhanced accumulation in cells and persistent stabilization of the cleavable complex [18-21]. Moreover, the recently described breast cancer resistance-associated protein (BCRP) does not recognize gimatecan. BCRP is important in cellular resistance to topotecan, irinotecan and mitoxantrone [22]. Gimatecan's potency is related to a larger number of DNA strand breaks compared to other camptothecin analogs, and it is particularly effective against some human tumor xenografts derived from adult tumors [19]. Finally, this drug is highly bioavailable by the oral route, which is of particular interest especially in pediatric patients, and is therefore suitable for prolonged daily administration.

The aim of the present study was to evaluate the antitumor activity and the pharmacokinetics of gimatecan given orally to pediatric tumor xenografts.

\section{Materials and methods}

\section{Drugs}

Gimatecan and SN-38 were kindly provided by Sigma-Tau, Pomezia, Rome, Italy. The drugs were dissolved in dimethyl

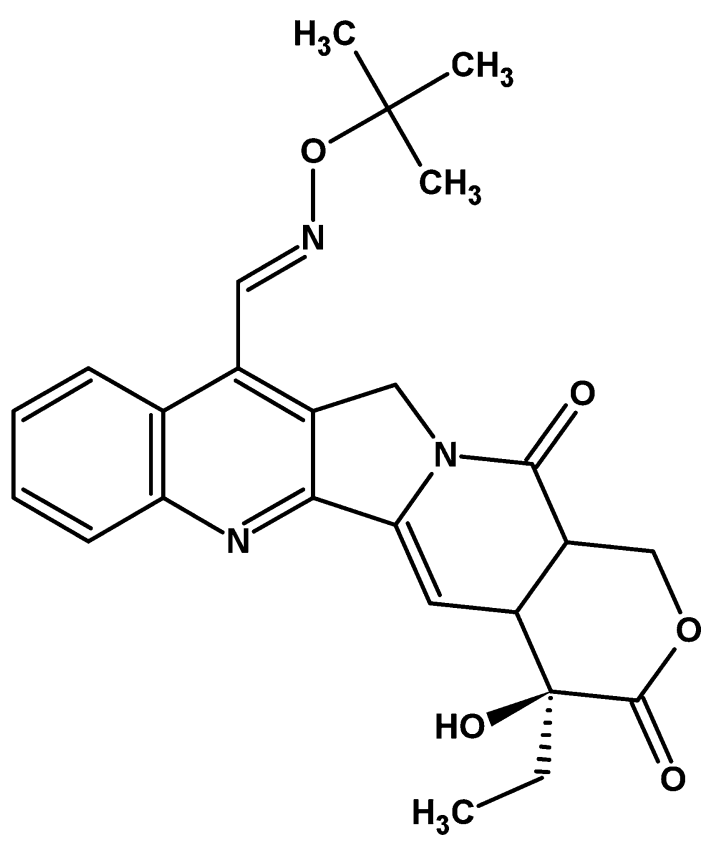

Fig. 1 Molecular structure of gimatecan sulfoxide (DMSO) to make the stock solutions $(10 \mathrm{mg} / \mathrm{ml}$ for gimatecan and $10 \mathrm{mM}$ for $\mathrm{SN}-38$ ), which were stored in small aliquots at $-20^{\circ} \mathrm{C}$, protected from light. Irinotecan (CPT-11, Campto ${ }^{\circledR}$, Pfizer Italia, Latina, Italy) for in vivo studies was dissolved in sterile distilled water.

Cell cultures and culture conditions

The human neuroblastoma cell lines (SK-N-DZ, SK-N$\mathrm{BE}(2) \mathrm{c})$ and human rhabdomyosarcoma TE-671 cells were purchased from the American Type Culture Collection (Rockville, MD, USA). Cells were maintained in RPMI 1640 medium, supplemented with $10 \%$ fetal calf serum (FCS), $2 \mathrm{mM} \mathrm{L-glutamine}$ and penicillin-streptomycin at $37^{\circ} \mathrm{C}$ in $5 \% \mathrm{CO}_{2}$.

\section{Cell sensitivity}

Cell sensitivity to gimatecan and SN-38 was assessed by 5day MTT assay. Cells were seeded at the appropriate density in 96-well plates, grown overnight and exposed to serial dilutions of the two drugs. After $72 \mathrm{~h}$ of incubation, $150 \mu \mathrm{g} /$ well MTT was added for $3 \mathrm{~h}$ at $37^{\circ} \mathrm{C}$. Formazan products were solubilized with $200 \mu \mathrm{l}$ of DMSO, and absorbance was detected at $540 \mathrm{~nm}$ (reference wavelength $690 \mathrm{~nm}$ ) using a microplate spectrophotometer. Cytotoxicity was assessed from dose-response curves as the $\mathrm{IC}_{50}$, which is the drug concentration of drug required to inhibit by $50 \%$ cell growth. All experiments were run in triplicate.

Mice

CD1 nu/nu male nude mice (Charles River, Calco, Italy) at 5 weeks of age were used. All animal investigations complied with the guidelines of the Istituto Superiore di Sanità (Rome, Italy) on experimental neoplasia in animals. The animals were maintained under specific pathogen-free conditions.

Pediatric xenografts and tumor growth

We evaluated the effects of gimatecan and CPT-11 in two human pediatric neuroblastoma cell lines (SK-N-DZ, SK$\mathrm{N}-\mathrm{BE}(2) \mathrm{c})$ and in the human rhabdomyosarcoma cell line (TE-671), growing as xenografts in nude mice; $20 \times 10^{6}$ cells were injected subcutaneously for SK-N-DZ and SK$\mathrm{N}-\mathrm{BE}(2) \mathrm{c}$ and $1.5 \times 10^{6}$ cells for TE-671 in both flanks. Two different schedules were used: qdx $5 / \mathrm{wx} 4 \mathrm{w}$ and q4dx4. In the first schedule, gimatecan was administered $0.25 \mathrm{mg} /$ $\mathrm{kg} / \mathrm{d}$ orally, whereas CPT-11 injected i.p. at $10 \mathrm{mg} / \mathrm{kg} / \mathrm{d}$; in the second schedule, gimatecan and CPT11 were administered, respectively, $1.25 \mathrm{mg} / \mathrm{kg} / \mathrm{d}$ and $50 \mathrm{mg} / \mathrm{kg} / \mathrm{d}$. Each control or drug-treated group consisted of $8-10$ mice. 
Tumor volumes were calculated by the formula: $d^{2} \times D / 2$, where $d$ and $D$ are, respectively, the shortest and the longest diameters.

The efficacy of drug treatment was assessed as percentage tumor volume inhibition (TVI\%) in treated $(\mathrm{T})$ versus control (C) mice according to the formula: $100-(\mathrm{T} / \mathrm{C} \times 100)$. $\log _{10}$ Cell Kill (LCK): $(\mathrm{T}-\mathrm{C}) / 3.32 \times \mathrm{DT}$ where $\mathrm{T}$ is the mean time (days) required for treated tumors and $\mathrm{C}$ for the control to reach an established volume (400 $\mathrm{mg})$, and DT is the mean Doubling Time of control tumors.

Complete regression (CR) was defined as tumor disappearance lasting at least 10 days during or after treatment $[6,21]$.

Student's test (two-tailed) was applied to compare in vivo tumor growth between treated and control groups; the differences were considered statistically significant at $P<0.05$. For statistical analyses, we randomly selected one of the bilateral tumors in each animal, because the growth and the responses to the treatment were similar in both flanks.

\section{Pharmacokinetic study}

Mice bearing neuroblastoma and rhabdomyosarcoma xenografts were treated orally with single or repeated (5 days) doses of $0.25 \mathrm{mg} / \mathrm{kg}$ of gimatecan. At different times $(0$, $0.25,0.5,1,2,4,8$ and $24 \mathrm{~h}$ ) after the single dose and after the last dose on day five, three mice per time point were sacrificed. The tumors were removed and the blood collected into heparinized tubes by cardiac puncture. Plasma was obtained by centrifugation at $3,000 \mathrm{rpm}$ at $4^{\circ} \mathrm{C}$ and stored frozen, together with the tumors, at $-20^{\circ} \mathrm{C}$ until analysis.

A highly specific and sensitive validated HPLC method was used to determine total gimatecan (lactone plus hydroxyacid form) and its major metabolite, the t-butyl hydroxy derivative in mouse plasma samples and tumors [23]. The method, originally developed in human plasma, is based on on-line column solid-phase extraction under acid conditions of the analytes from plasma after addition of the internal standard (IS, 20-O-butirryl-camptothecin) chromatographic separation under isocratic conditions and fluorimetric detection. The limit of quantification is $0.5 \mathrm{ng} / \mathrm{ml}$ for both analytes.

Tumors were homogenized and extracted in four volumes (p:v) of $0.1 \mathrm{~N} \mathrm{HCl}: \mathrm{CH}_{3} \mathrm{CN}$ (1/10), centrifuged, the organic phase was mixed with $50 \mathrm{ng}$ of IS and diluted 1:1 with $0.1 \% \mathrm{HCOOH}: \mathrm{CH} 3 \mathrm{CN}$ (1:1). After centrifugation for $10 \mathrm{~min}$ at $4,000 \mathrm{rpm}$, portions of the supernatants were injected for HPLC analysis.

In a second experiment to determine the presence of the intact lactone form of gimatecan, a modification of the present HPLC method was introduced, and the intact lactone form was assayed in plasma of CD1 male mice [23]. Analysis was carried out without acidification of the plasma samples and consisted of the addition of $100 \mu \mathrm{l}$ of bi-distilled water to $100 \mu \mathrm{l}$ of plasma and of IS. Chromatographic separation was achieved under neutral $\mathrm{pH}$ conditions with a mobile phase of $50 \%$ acetonitrile in bi-distilled water. Samples were quantified using the same standard calibration curve and quality control samples prepared for the assay of total gimatecan with extraction under acid conditions, but using neutral $\mathrm{pH}$ chromatographic separation with a mobile phase of 50\% acetonitrile in bi-distilled water.

The analytical reference standard of gimatecan (batch No 532/68) was provided by Antibioticos S.p.A. The analytical reference standard of 20-O-butirryl-camptothecin (batch No EM/1/1) used as IS was provided by Sigma-Tau.

\section{Results}

In vitro growth inhibition

The cytotoxic effects of gimatecan and SN-38 were evaluated in vitro by the MTT assay after 3 days exposure. As shown in Table 1, gimatecan was substantially more potent than SN-38 in all cell lines. The neuroblastoma cell lines SK-N-DZ and SK-N-(BE)2c showed particularly high sensitivity to gimatecan in comparison with the rhabdomyosarcoma cell line (up to 68- and 13-fold). The SN-38 concentrations required to inhibit cell growth by $50 \%$ in neuroblastoma cells were 900 and $600 \mathrm{nM}$ for SK-N-DZ and SK-N-(BE)2c, and the $\mathrm{IC}_{50}$ for TE-671 cells was $2100 \mathrm{nM}$.

Xenograft growth inhibition

We compared the effects of gimatecan (p.o.) and irinotecan on pediatric tumors from neuroblastoma (SK-N-DZ and SK-N-BE(2)c) and rhabdomyosarcoma (TE-671) (Fig. 2). Two different schedules were used: qdx5/wx4w and q4dx4. With both schedules, the maximum tolerated doses (MTD) were $5 \mathrm{mg} / \mathrm{kg}$ for gimatecan and $200 \mathrm{mg} / \mathrm{kg}$ for CPT- 11 .

Table 1 Five-day MTT assay on a panel of different tumor cell lines treated with gimatecan and SN-38

\begin{tabular}{lccc}
\hline Drug & \multicolumn{1}{l}{$\mathrm{IC}_{50}(\mathrm{nM})$} & & \\
\cline { 2 - 4 } & \multicolumn{1}{c}{$\mathrm{TE}-671$} & SK-N-DZ & SK-N-(BE)2c \\
\hline Gimatecan & $115 \pm 21$ & $1.7 \pm 0.4$ & $9.1 \pm 1.8$ \\
SN-38 & $2100 \pm 0.8$ & $900 \pm 1.1$ & $600 \pm 0.3$ \\
\hline
\end{tabular}

$\mathrm{IC}_{50}$ Drug concentration required for $50 \%$ reduction in cell growth when compared with untreated controls. The data are an average of at least three independent experiments 

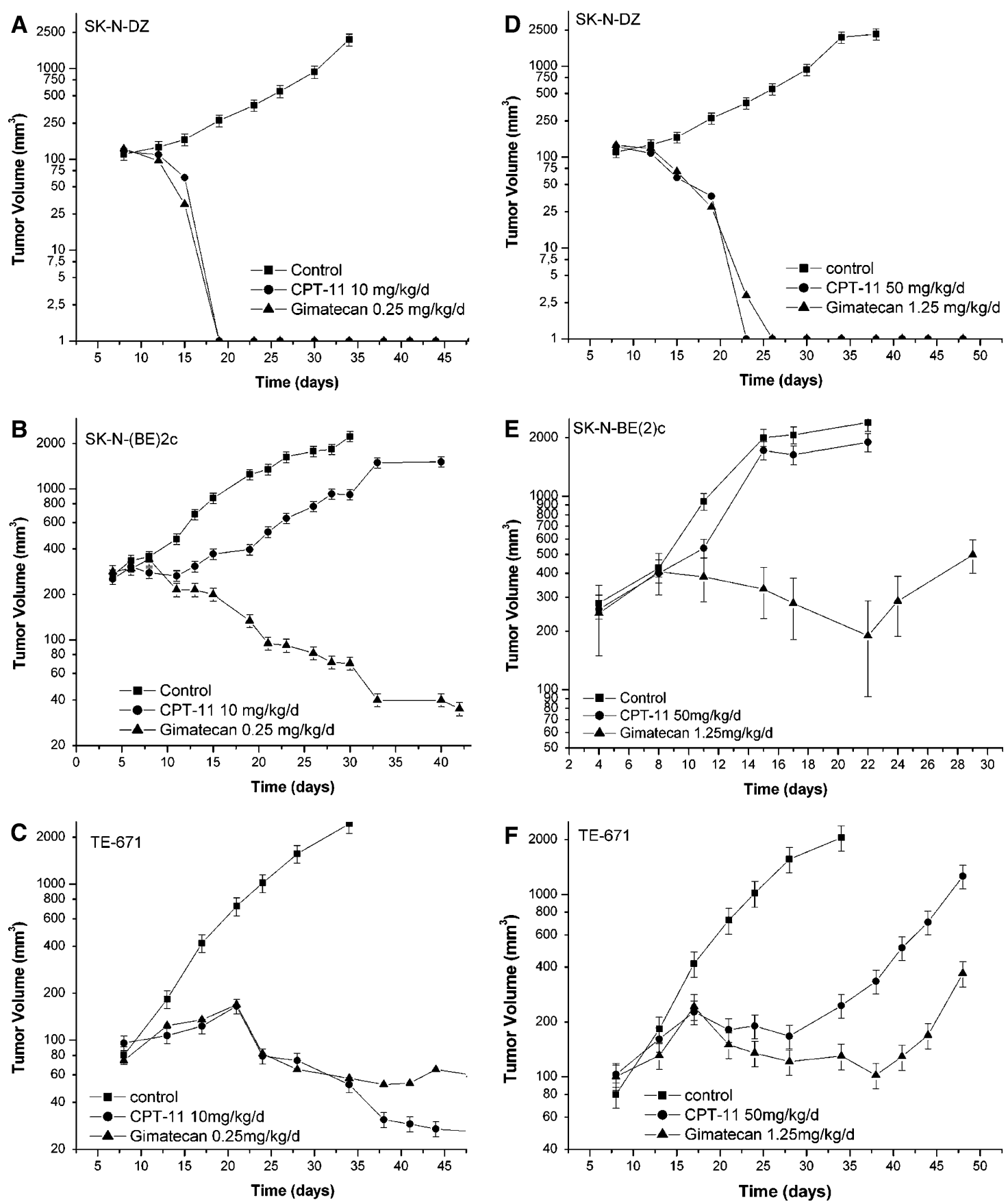

Fig. 2 Growth curves of tumor xenografts in mice treated with gimatecan or CPT-11 by two different schedules. Tumor cells were injected s.c. into the flank of the nude mice as described in Materials and methods. a, b and $\mathbf{c}$ qdx $5 / \mathrm{w} \times 4$ schedule: gimatecan was given orally at the

dose of $0.25 \mathrm{mg} / \mathrm{kg} / \mathrm{d}$, CPT- 11 i.p. at $10 \mathrm{mg} / \mathrm{kg} / \mathrm{d}$. c, d and e q $4 \mathrm{dx} 4$ schedule: gimatecan was given orally at the dose of $1.25 \mathrm{mg} / \mathrm{kg} / \mathrm{d}$, CPT-11 i.p. at $50 \mathrm{mg} / \mathrm{kg} / \mathrm{d}$; Symbols: Filled square control; Filled circle CPT-11; Filled triangle gimatecan. Bars, SD

The antitumor activity of the drugs is shown in Fig. 2. With the qdx $5 / \mathrm{wx} 4 \mathrm{w}$ schedule, gimatecan produced a statistically significant tumor regression in all tumor xenografts $(P$ at least $<0.008$ ). In SK-NDZ model, a complete disappear of tumor was observed in $100 \%$ of mice. As no tumor regrowth was observed up to 15 days from the end of treatment, this was considered a CR. In a prolonged observation

up to 72 days, no tumor regrowth was observed in the two NB models, whereas in the rhabdomyosarcoma xenograft, there was a high incidence of tumor regrowth (data not shown).

CPT-11 induced regression of the tumor on SK-N-DZ and TE-671 xenografts, but was completely ineffective against SK-N-(BE)2c xenografts. Consistently with the 
gimatecan data, regression was maintained in the SK-N-DZ model, but not in the TE-671 after 72 days of observation (data not shown).

With the $\mathrm{q} 4 \mathrm{dx} 4$ schedule gimatecan completely inhibited the tumor growth only of the SK-N-DZ xenografts, and the TE-671 and SK-N-(BE)2c xenografts were less sensitive, showing significant $(P<0.04)$ but not complete tumor regression (TVI\% and LCK: 78 and 2.3 for TE-671; 92 and 1.1 for SK-N-(BE)2c). CPT-11 had different effects in the three models: a complete response was achieved only on SK-N-DZ tumor xenografts, and TE-671 xenografts regrew at the end of the treatment. It had no antitumoral activity on SK-N-(BE)2c xenografts.

Gimatecan was generally well tolerated, with no signs of acute or delayed toxicity. An approximately $10-15 \%$ reduction in body weight was observed at the end of the treatment (data not shown), but this was reversible upon cessation of treatment.

\section{Pharmacokinetics of gimatecan}

Figures 3 and 4 show the plasma levels and the tumor distribution of gimatecan in mice bearing SK-N-DZ neuroblastoma and TE-671 rhabdomyosarcoma after oral doses of $0.25 \mathrm{mg} / \mathrm{kg}$. The main pharmacokinetic parameters are listed in Table 2.
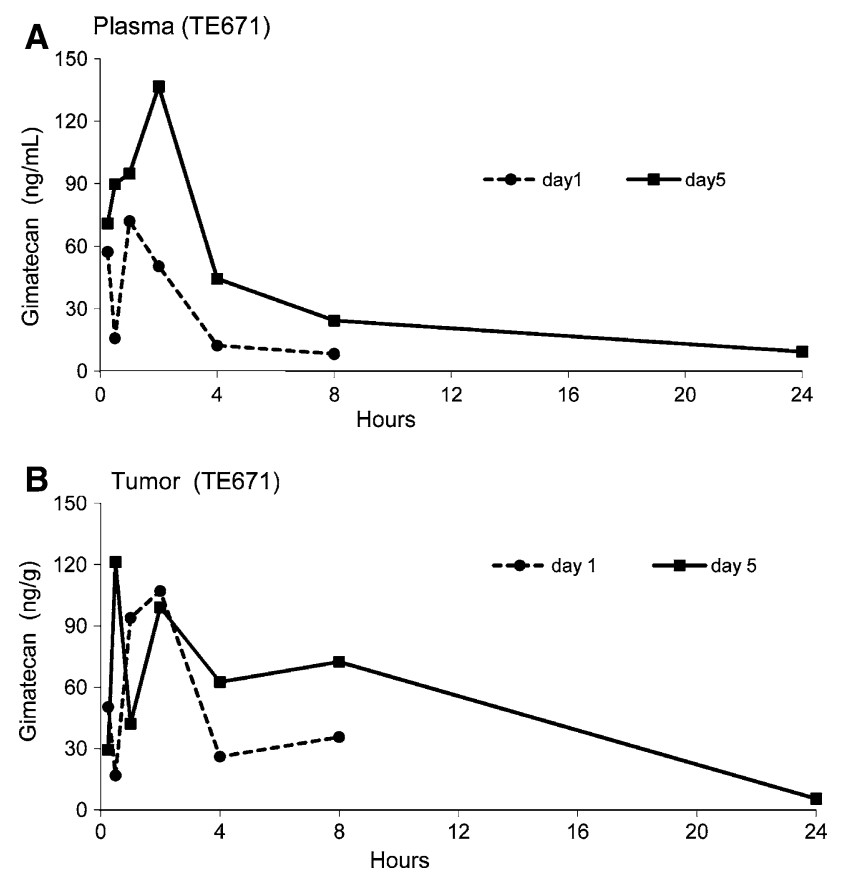

Fig. 3 Plasma and tumor levels of gimatecan after single or repeated 5 days oral doses of $0.25 \mathrm{mg} / \mathrm{kg}$ in nude mice with TE-671 rhabdomyosarcoma. At different times (0, 0.25, 0.5, 1,2, 4, 8 and $24 \mathrm{~h})$ after the single and the last dose on day five, three mice per time point were sacrificed
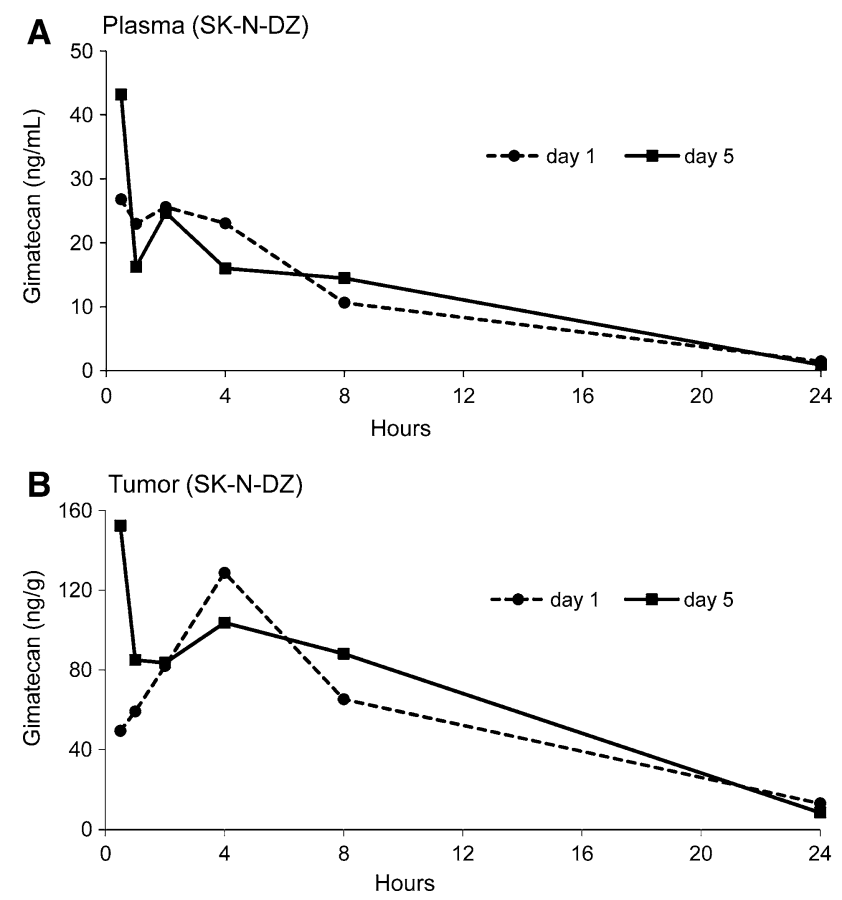

Fig. 4 Plasma and tumor levels of gimatecan after single or repeated 5 days oral doses of $0.25 \mathrm{mg} / \mathrm{kg}$ in nude mice with SK-N-DZ neuroblastoma. At different times $(0,0.25,0.5,1,2,4,8$ and $24 \mathrm{~h})$ after the single and the last dose on day five, three mice for time point were sacrificed

Table 2 Main plasma pharmacokinetic parameters and tumor distribution of gimatecan in SK-N-DZ and TE-671 bearing mice

\begin{tabular}{lrrrll}
\hline Pharmacokinetic & \multicolumn{2}{l}{ SK-N-DZ } & & & TE-671 \\
\cline { 2 - 3 } \cline { 6 - 6 } parameters & Day 1 & Day 5 & & Day 1 & Day 5 \\
\hline AUCexp (ng h/ml) & 256.4 & 271.4 & & 269.3 & 778.3 \\
T1/2 last (h) & 5.2 & 4.6 & - & 9.5 \\
Cmax (ng/ml) & 26.8 & 43.2 & 72.1 & 136.7 \\
T max (h) & 0.5 & 0.5 & 1 & 2 \\
Tumor AUC & 1339.0 & 1576.9 & & 399.7 & 1283.2 \\
Tumor Cmax & 128.7 & 152.5 & & 107.1 & 121.3 \\
Ratio & 5.2 & 5.7 & & 1.5 & 1.5 \\
\hline
\end{tabular}

AUC: area under the concentration-time curve from 0 to infinity T1/2 last: apparent terminal half-life

Cmax: maximal plasma concentration

Ratio: tumor AUC/plasma AUC

After the single dose, gimatecan achieved peak plasma concentration and AUC of $72.1 \mathrm{ng} / \mathrm{ml}$ and $269.3 \mathrm{ng} / \mathrm{ml} \times \mathrm{h}$ in mice with sarcoma and $26.8 \mathrm{ng} / \mathrm{ml}$ and $256.6 \mathrm{ng} / \mathrm{ml} \times \mathrm{h}$ in mice with neuroblastoma. In both cases, the drug was undetectable in plasma at $24 \mathrm{~h}$.

Gimatecan achieved higher and more persistent concentrations in the tumor tissue than in plasma and distributed more extensively in SK-N-DZ tumor than in TE-671 tumor, with ratios of tumor AUC to plasma AUC of 5.2 and 1.5 for 


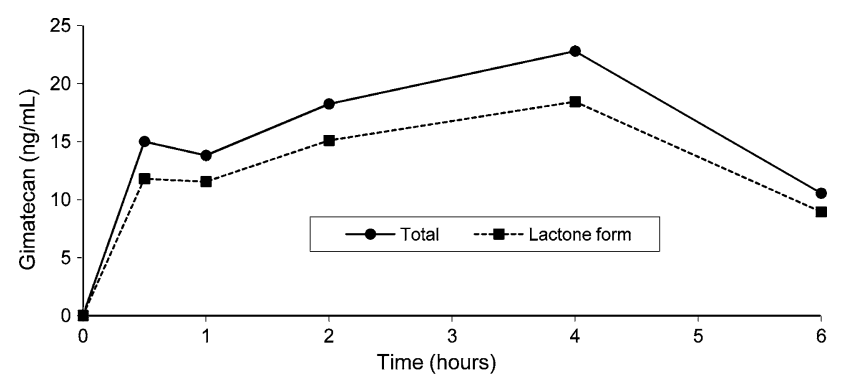

Fig. 5 Gimatecan plasma levels (total and lactone form) in mice treated with $0.25 \mathrm{mg} / \mathrm{kg}$ of gimatecan

SK-N-DZ and TE-671 bearing mice, respectively. In the SK-N-DZ tumor, these concentrations were at all times $\geq 10 \mathrm{ng} / \mathrm{g}(22 \mathrm{nM}), 12$ times the $\mathrm{IC}_{50}$ (Table 1), whereas in TE-671 tumor, gimatecan achieved concentrations superior to the $\mathrm{IC}_{50}(115 \mathrm{nM})$ only up to $2 \mathrm{~h}$.

After the repeated treatment, plasma levels of gimatecan were higher in both groups of mice than after a single dose. The drug disappeared from plasma with a half-life of 5$9.5 \mathrm{~h}$, being still detectable at $24 \mathrm{~h}$ in TE- 671 mice, with accumulation in tumor tissue. On day five, the gimatecan AUC remained high $(1576.9 \mathrm{ng} / \mathrm{ml} \times \mathrm{h})$ in SK-N-DZ tumor, and rose threefold in TE-671 tumor (from 399.7 to $1283.2 \mathrm{ng} / \mathrm{ml} \times \mathrm{h}$ ) achieving concentrations in this tumor in the range of 120-200 $\mathrm{nM}$, superior to the $\mathrm{IC}_{50}$ (Table 1).

In a separate experiment, using a modification of the HPLC method used to analyze total gimatecan [23], the intact lactone form was assayed in plasma of mice for $0-6 \mathrm{~h}$ after a single dose of $0.25 \mathrm{mg} / \mathrm{kg}$ of gimatecan. Figure 5 compares the plasma levels of total gimatecan and the intact lactone; gimatecan was detected in plasma almost completely as the intact lactone form (more than 80\%). This is particularly important as this form is responsible for the pharmacological activity of the camptothecins.

\section{Discussion}

The present study found that gimatecan was significantly effective in the treatment of pediatric neuroblastoma and rhabdomyosarcoma grown s.c. in nude mice. On these tumor models, gimatecan was more effective than CPT-11, used for comparison in inhibiting cell proliferation and tumor growth in vitro and in vivo.

In vitro gimatecan had significantly higher antiproliferative potency than SN-38 on all three cell lines. SK-N-DZ showed higher sensitivity to gimatecan than the rhabdomyosarcoma cell line (up to 68-fold), whereas the sensitivity to $\mathrm{SN}-38$ was similar in both cell lines. These data are comparable with those of a previous study on a panel of neuroblastoma cell lines, despite the low intracellular accumulation of gimatecan [19].
Gimatecan also showed superior antitumor activity in vivo with two different treatment schedules that appeared well tolerated by mice, with only a $10-15 \%$ reduction in body weight at the end of the treatment.

With the $\mathrm{qd} \times 5 / \mathrm{w} \times 4 \mathrm{w}$ treatment, gimatecan produced a complete response in all tumor xenografts. CPT-11 induced complete tumors response in SK-N-DZ and TE671 xenografts, but was ineffective against SK-N-(BE)2c. With the $\mathrm{q} 4 \mathrm{dx} 4$ schedule, gimatecan completely inhibited the growth of SK-N-DZ tumors xenografts, whereas TE671 and SK-N-(BE)2c xenografts were less sensitive, though they still showed significant regression. The effects of CPT-11 were different in the three models, with complete regression only on SK-N-DZ, whereas TE-671 xenografts re-grew at the end of the treatment, and no antitumoral activity was observed on SK-N-(BE)2c xenografts.

The in vivo results were supported by pharmacokinetic data showing that gimatecan reached high levels in plasma and in tumor tissues at concentrations that are potentially active in vitro. In the most responsive tumor, the neuroblastoma SK-N-DZ, gimatecan achieved concentrations in tumor tissue that were at least from 2 to 12 times the $\mathrm{IC}_{50}$ and were measurable throughout the 24-h sampling on both days ( 1 and 5) of the pharmacokinetic study. In the other tumor model (TE-671), that was less sensitive in vitro and less responsive in vivo, gimatecan reached concentrations higher than the $\mathrm{IC}_{50}$, measurable throughout the 8-h sampling only on day 5 . It is also to notice that the AUCs we found in mice were three-sixfold lower than the AUC previously reported by Sessa et al. relative to a dose of $4.5 \mathrm{mg} /$ $\mathrm{m}^{2}$ (daily $0.9 \mathrm{mg} / \mathrm{m}^{2}$ ) that correspond to the MTD in humans [24].

We found that gimatecan had some distinct pharmacological properties, in particular the unique stability of the lactone form in plasma (the form generally considered active as a DNA topoisomerase I poison) that accounts for $>80 \%$ of the total in mice, differently from the other clinically used camptothecins, where $30-70 \%$ of the lactone is present in plasma [17].

In conclusion, gimatecan showed high antitumor activity in several pediatric tumor models, supported by pharmacokinetic evidence of high oral disposition of the drug, present largely as the lactone form, with high and lasting distribution in tumor tissue. This particular pharmacological behavior suggests that gimatecan might be of interest in the treatment of pediatric solid tumors.

\section{References}

1. Pommier Y (2006) Topoisomerase I inhibitors: camptothecin and beyond. Nat Rev Cancer 6:789-802 
2. Liu LF (1989) DNA topoisomerase poison as antitumor drugs. Ann Rev Biochem 58:351-375

3. Shimada Y, Yoshino M, Wakui A, Nakao I, Futatsuki K, Sakata Y et al (1993) Phase II study of CPT-11, a new camptothecin derivative, in metastatic colorectal cancer. CPT-11 gastrointestinal cancer study group. J Clin Onc 11:909-913

4. Kambe M, Wakui A, Nakao I, Futatsuki K, Sakata Y, Yoshino M (1993) A late phase of study of irinotecan (CPT-11) in patients with advanced gastric cancers. Pro Am Soc Clin Oncol 12:198

5. Rocha Lima CM, Eckardt JR, Leong SS, Sherman CA, Perkel JA, Putman T et al (1999) Single-agent gemcitabine and gemcitabine/ irinotecan combination (irimogem) in non-small cell lung cancer. Semim Oncol 26:43-50

6. Thompson J, Zamboni WC, Cheshire PJ, Lutz L, Luo X, Li Y et al (1997) Efficacy of systemic administration of irinotecan against neuroblastoma xenograft. Clin Cancer Res 3:423-431

7. Rivory LP (1996) Irinotecan (CPT-11): a brief overview. Clin Exp Pharmacol Physiol 23:1000-1004

8. Santos A, Calvet L, Terrier-Lacombe MJ, Larsen A, Bérnard J, Pondarré $\mathrm{C}$ et al (2004) In vivo treatment with CPT-11 leads to differentiation of neuroblastoma xenografts and topoisomerase I alterations. Cancer Res 64:3223-3229

9. Houghton PJ, Cheshire PJ, Hallman JC, Bissery MC, MathieuBoué A, Houghton JA (1993) Therapeutic efficacy of the topoisomerase I inhibitor 7-ethyl-10-(4-[1-piperidino]-1-piperidino)-carbonyloxy-camptothecin against human tumor xenografts: lack of cross-resistance in vivo in tumors with acquired resistance to the topoisomerase I inhibitor 9-dimethylaminomethyl-10-hydroxycamptothecin. Cancer Res 53:2823-2829

10. McGuire WP, Blessing JA, Bookman MA, Lentz SS, Dunton CJ (2000) Topotecan has substantial antitumor activity as first-line salvage therapy in platinum-sensitive epithelial ovarian carcinoma: a gynecologic oncology group study. J Clin Oncol 18:1062-1067

11. Tubergen DG, Stewart CF, Pratt CB, Zamboni WC, Winick N, Santana VM et al (1996) Phase I trial and pharmacokinetic (PK) and pharmacodynamics (PD) study of topotecan using a five-day course in children with refractory solid tumors: a pediatric oncology group study. J Pediatr Hematol Oncol 18:352-361

12. Santana VM, Furman WL, Billups CA, Hoffer F, Davidoff AM, Houghton PJ et al (2005) Improved response in high-risk neuroblastoma with protracted topotecan administration using a pharmacokinetically guided dosing approach. J Clin Oncol 23:4039-4047
13. Thomas CJ, Rahiner NJ, Hecht SM (2004) Camptothecin: current perspectives. Bioorg Med Chem 12:1585-1604

14. Burke TG, Munshi CB, Mi Z, Jiang Y (1995) The important role of albumin in determining the relative human blood stabilities of the campthotecin anticancer drug. J Pharm Sci 84:518-519

15. Beretta GL, Zunino F (2007) Relevance of extracellular and intracellular interactions of camptothecins as determinants of antitumor activity. Biochem Pharmacol 74:1437-1444

16. Beretta L, Perego P, Zunino F (2006) Mechanism of cellular resistance to champtothecins. Curr Med Chem 13:3291-3305

17. Garcia-Carbone R, Supko JG (2002) Current perspectives on the clinical experience, pharmacology, and continued development of the camptothecin. Clin Cancer Res 8:641-661

18. Perego P, Ciusani E, Gatti L, Carenini N, Corna E, Zunino F (2006) Sensitization to gimatecan-induced apoptosis by tumor necrosis factor-related apoptosis inducing ligand in prostate carcinoma cells. Biochem Pharm 71:791-798

19. Di Francesco AM, Riccardi A, Barone G, Rutella S, Meco D, Frapolli R et al (2005) The novel lipophilic camptothecin analogue gimatecan is very active in vitro in human neuroblastoma: a comparative study with SN38 and topotecan. Biochem Pharmacol 70:1125-1136

20. De Cesare M, Pratesi G, Veneroni S, Bergottini R, Zunino F (2004) Efficacy of the novel camptothecin gimatecan against orthotopic and metastatic human tumor xenograft models. Clin Cancer Res 10:7357-7364

21. De Cesare M, Pratesi G, Perego P, Carnini N, Tinelli S, Merlini L et al (2001) Potent antitumor activity and improved pharmacological profile of ST1481, a novel 7-substituted camptothecin. Cancer Res 61:7189-7195

22. Perego P, De Cesare M, De Isabella P, Carenini N, Beggiolin G, Pezzoni G et al (2001) A novel 7-modified camptothecin analog overcomes breast cancer resistance protein-associated resistance in a mitoxantrone-selected colon carcinoma cell line. Cancer Res 61:6034-6037

23. Pace S, Capocasa F, Tallarico C, Frapolli R, Zucchetti M, Longo A (2009) Determination of total and lactone form of a new camptothecin derivative Gimatecan (ST1481) and its metabolite ST1698 in human plasma by high-performance liquid chromatography with fluorimetric detection. J Pharm Biomed Anal 50:507-514

24. Sessa C, Cresta S, Cerny T, Baselga J, Rota Caremoli E, Malossi A et al (2007) Concerted escalation of dose and dosing duration in a phase I study of the oral camptothecin gimatecan (ST1481) in patients with advanced solid tumors. Ann Oncol 18:561-568 